\begin{tabular}{c} 
International Journal of Engineering \& Technology, 7 (3) (2018) $1482-1490$ \\
International Journal of Engineering \& Technology \\
SPC \\
Website: $\begin{array}{c}\text { www.sciencepubco.com/index.php/IJET } \\
\text { doi: } 10.14419 / \text { ijet. } 7 \text { 7i3.14084 } \\
\text { Research paper }\end{array}$ \\
\hline
\end{tabular}

\title{
Automatic detection and classification of cardiac arrhythmia using neural network
}

\author{
N. N. S. V Rama Raju ${ }^{1}$, V. Malleswara $\operatorname{Rao}^{1}$, I. Srinivasa $\operatorname{Rao}^{1}$ \\ ${ }^{1}$ Dept. of ECE, GITAM University, Visakhapatnam \\ *Corresponding author E-mail: ramarajupro@gmail.com
}

\begin{abstract}
This paper proposes a Neural Network classifier model for the automatic identification of the ventricular and supraventricular arrhythmias cardiac arrhythmias. The wavelet transform (DWT) and dual tree complex wavelet transform (DTCWT) is applied for QRS complex detection. After segmentation both feature of DWT and DTCWT is combined for feature extraction, statistical feature has been calculated to reduce the overhead of classifier. An adaptive filtering with the soft computed wavelet thersholding to the signals before the extraction is done in pre-processing. Different ECG database is considered to evaluate the propose work with MIT-BIH database Normal Sinus Rhythm Da-tabase (NSRD), and MIT-BIH Supraventricular Arrhythmia Database (svdb) .The evaluated outcomes of ECG classification claims $98-99 \%$ of accuracy under different training and testing situation.
\end{abstract}

Keywords: Cardiac Arrhythmias; DTCWT; DWT; MIT-BIH; Neural Network; QRS.

\section{Introduction}

Ventricular tachycardia which are dangerous and the ECG signal represents the electrical activity of the need for immediate therapy with a heart. The ECG shown in Figure 1 represents the electrical record of heart activity, where each heartbeat is shown by a series of electric waves categorized by peaks and valleys [1].

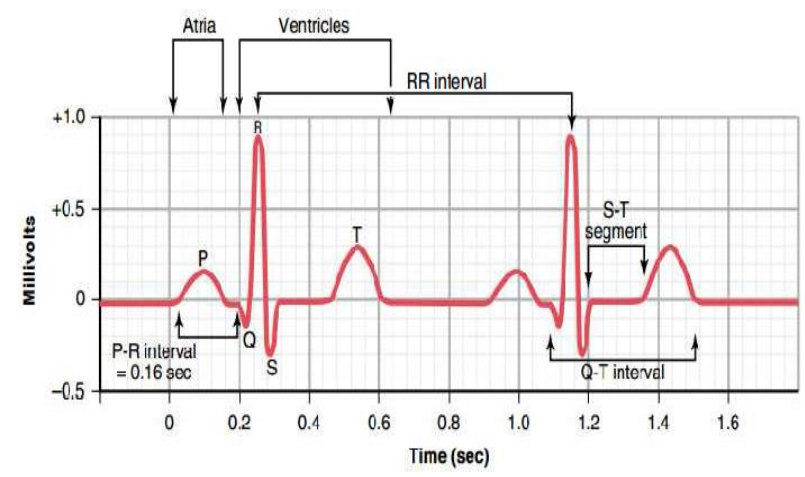

Fig. 1: Components of a Normal Electrocardiogram (ECG) [1].

The importance of the electrocardiographic (ECG) signal is well known for the diagnosis of a multitude of heart diseases, both through visual inspection and through automatic inspection techniques. As in the analysis of any other signal, the quality and quantity of information obtained will depend on properties such as the signal-to-noise ratio (SNR), the resolution of the analog-to-digital converter, the sampling frequency, etc. For this reason and prior to its automated treatment, it is extremely important that the signal provides us with information with adequate quality, especially since it is taken into account that the diagnosis of certain serious cardiovascular diseases depends on it. On the other hand, in the acquisition of long-term ECG, records (called Holter) the number of beats to be examined by the medical team may be several thousand, which makes visual inspection of such a signal in a laborious and complex task. In fact, the Holters correspond to ambulatory records of 24 or 48 hours, which are then transmitted to the analysis team at a speed of 60 to 120 times the acquisition speed [2]. Then the significant events are extracted, often manually. The automatic analysis of these signals constitutes an interesting field of investigation, due to the difficulty of its manual analysis. However, the methods used so far to carry out this task, do not provide sufficiently satisfactory results and it is necessary to review the automatically generated results to avoid the loss of beats with important information. In our case, we intend to analyze different clustering techniques applied to ECG Holter beats to obtain a grouping by types of the different beats that appear in an electrocardiographic signal. Each of the groups obtained should be completely homogenous so that it only contains identical beats from the diagnostic point of view, so that the cardiologist should only examine one beat in each group to determine the patient's pathology, significantly reducing the amount of data to be inspected.

\subsection{Interpretation of ECG component waves}

During the phases of depolarization and myocardial repolarization and on the ECG, a series of electrocardiographic waves appear. Distortion of the ECG component waves between the waves are called intervals while the ECG portions that appear between the deflections are called segments. An ECG period belonging to a healthy individual comprises a $\mathrm{P}$ wave, a QRS complex, a $\mathrm{T}$ wave and a $\mathrm{U}$ wave, as shown in Figure 2. 


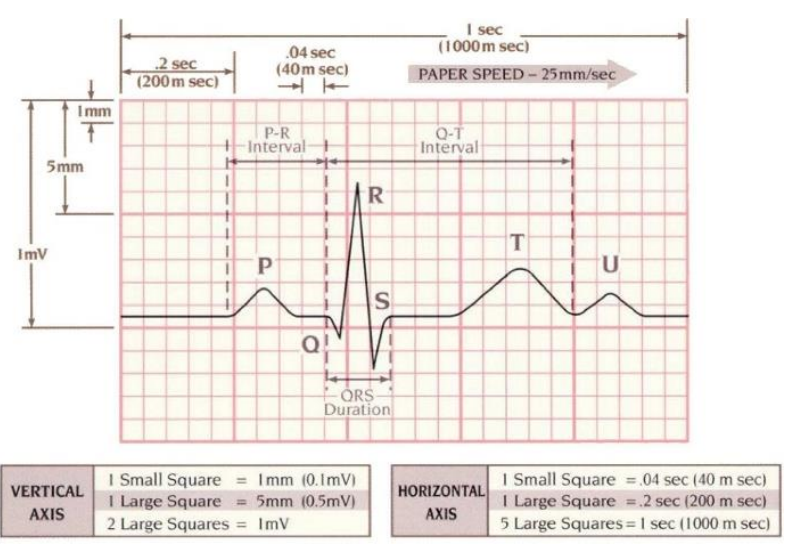

Fig. 2: Ideal Model of the Cardiac Cycle [3].

Within the ECG of a cardiac cycle we can distinguish the following waves, deflections and segments:

\subsubsection{P wave}

It represents the depolarization of the atrium (which involves contraction or atrial systole). Its normal duration is $0.1 \mathrm{~s}$. The shape of this wave depends on the location of the electrodes (shunt). An increase in the voltage of this wave indicates an atrial abnormality The absence of this wave occurs at a sinus node stop and at the sinoatrial SA block (situation in which the sinus node is depolarized but the impulse is not transmitted to adjacent atrial cells).

\subsubsection{QRS complex}

It characterizes the depolarization of the ventricles (which means contraction or ventricular systole). It consists of the waves $Q, R$ and $\mathrm{S}$, although other variants called R0 and S0 may appear. Its approximate duration is $100 \mathrm{~ms}$.

\subsubsection{T wave}

Involves the repolarization of the ventricles (ventricular relaxation or diastole). The normal $\mathrm{T}$ wave is asymmetrical in its branches and is rounded at its apex. The slope of the initial branch is softer than that of the terminal branch. Anomalies in this wave may indicate primary (but not necessarily) primary heart disease. It can also produce hydro electrolytic balance disorders.

\subsubsection{U wave}

Its physiological origin is unclear, although it may be due to repolarization of the Purkinje system. It has an abnormal shape when potassium disorders occur.

\subsubsection{PR segment}

It corresponds to the isoelectric line defined from the start of the $\mathrm{P}$ wave (start of atrial deflection) to the initial deflection of the QRS complex (onset of ventricular deporalization). The normal duration of this segment is between 0.12 and $0.21 \mathrm{~s}$, a variation that depends on the heart rate.

\subsubsection{ST segment}

It is the interval between the end of the QRS complex and the start of the $\mathrm{T}$ wave. It represents the time during which the ventricles remain in the activated state and ventricular repolarization can be initiated. Normally the ST segment is isoelectric, although it can also appear slightly deviated. A high deviation often represents myocardial infarction, acute pericarditis, or myocarditis.

\subsubsection{PP interval}

Corresponding to the time elapsed between the beginning of the $\mathrm{P}$ wave and the beginning of the next $P$ wave.

\subsection{8. $R R$ interval}

Corresponding to the time elapsed between the $\mathrm{R}$ wave of a $\mathrm{QRS}$ complex and the $\mathrm{R}$ wave of the following QRS complex.

\subsubsection{QRS interval}

Elapsed time between the start of a $\mathrm{Q}$ wave and the end of the $\mathrm{S}$ wave within the same QRS complex. It is an indicator of intraventricular conduction time. The upper limit of normal interval of the QRS complex is less than $0.12 \mathrm{~s}$. Duration greater than $0.12 \mathrm{~s}$ means that the pulse was initiated from the AV node.

Strategies for investigating the ECG signal until a couple of years prior depended on time domain techniques. In any case, this is not generally adequate to examine every single attribute of ECG signals. In this way, representation in the frequency domain of a signal is essential. The FFT (Fast Fourier Transform) method can be connected, be that as it may, the constraint of this approach neglects to give information on the exact location of frequency components after some time. As the frequency substance of the ECG fluctuates after some time, the requirement for a detailed explanation of the frequency spectrum of the ECG at its temporal location becomes necessary.

This legitimizes the utilization of time-frequency representation to the quantitative parts of electrocardiology. The method accessible for this purpose is the Short-time Fourier transform (STFT), in spite of the fact that the primary disadvantage of this STFT is that its accuracy in the time-frequency domain is not ideal. In the middle of the several time-frequency transformations, the Wavelet Transform (WT) ends up being straightforward and more accurate.

The Wavelet Transform depends on an arrangement of examining wavelets or mother wavelet that permits the decomposition of an ECG signal into a set of coefficients [4]. Every single wavelet family has its location, duration time and frequency bandwidth. The Wavelet coefficients ensuing from the WT resembles to a degree of the ECG components for a certain time period and bandwidth fragment.

\section{Literature review}

The minutes, marked on the ECG by the number of changes concerning the QRS rhythm or frequency are cardiac arrhythmias (fatal diseases) and subject of this work. For clinical and technological problem highlighting arrhythmias, but they defy by (means and approaches) [5 - 9]. They are varied and Often depends on the point of view of the cardiac arrhythmias by the irregularity of the beats [7]. Depending on [10-11], it is possible to define premature ventricles [12 - 15] with cardiac arrhythmias as a group of different parameters, approaches and results. These parameters can be morphological, temporal such as [16 - 19] or statistical or both [20]. In [21] only the RR interval is used to search for several arrhythmias. On the other hand, various classification techniques have been proposed in the past years to classify the arrhythmic beats in normal or abnormal (arrhythmic) beats according to different rhythms [17], [22]. In [23], authors used network flow classification procedures to characterize the rhythms. In the majority of cases, the parameters like width, shape and amplitude are measured around the QRS complex and the dominant parameter [7] is the detection of the $\mathrm{R}$ wave. A number of significant parameters can be established to classify the arrhythmias present in the QRS complex. Inspired by several works cited, we propose a method by neural networks and wavelet transform (WT) which have very interesting adaptation properties. In the articles [24 - 27] successfully conducted many works using wavelets for the contribution of the ECG signal.

The identification of cardiac pathologies can be done with nonlinear array analysis of artificial neural network (ANN). The literature is rich in works that have exploited the advantages of ANN to automatically detect or classify cardiac arrhythmias such as [27 - 34]. Our results are estimated with MIT-BIH Data Arrhythmias of ECG recordings for detecting the pathologies like Extra ventricular (V), 
Extra supraventricular systolic (S), Fibrillation ventricular flutter $(\mathrm{V})$, ventricular tachycardia (VT) and ventricular bradycardia $(\mathrm{Br})$

\section{System model}

A bibliographical review of the methods and techniques used in the pre-processing, feature extraction, segmentation, as well as the sources related to the reduction of characteristics related to the stages of the ECG signal analysis process is discussed.

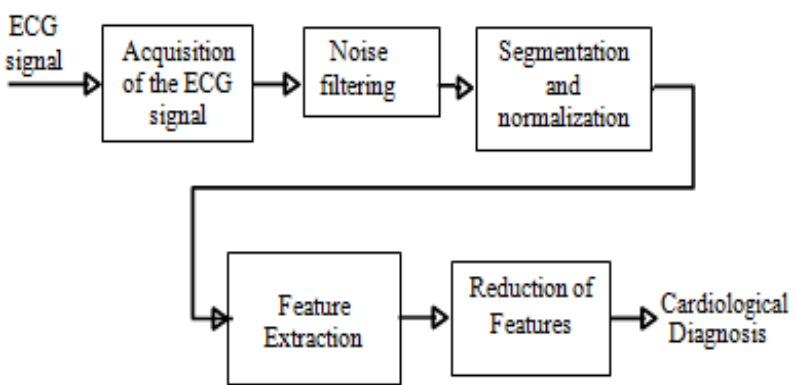

Fig. 3: General Process Developed in the Analysis of the Beats of an ECG Signal.

\subsection{ECG signal pre-processing}

The different noises that stain an ECG signal are considered undesirable, and can alter more or less the clinical information. In addition, the difficulties in detecting QRS complexes reside essentially in the great variability of the signal shape and the presence in the ECG of these unnecessary noises of various origins. It is therefore important to know what types of noise can contaminate an ECG electrocardiogram signal.

\subsubsection{Types of noise present in the ECG signal}

During the acquisition of the ECG signal, undesirable events called artifacts may appear on the electrocardiographic tracing. The problem is often posed during automatic signal processing, where the presence of these noises can lead to errors in the diagnosis. These noises can be categorized according to their origins into two broad categories, technical noises and physical noises.

\subsubsection{Technical noises}

Technical noises are noises caused by the material used during recording.

\subsubsection{Physical noise}

Physical noises are artefacts generated by either the electrical activities of the human body such as muscular contractions or movements during breathing.

\subsubsection{Noise cancellation}

Details coefficients is responsible for unwanted oscillations in the ECG signal. Thus two techniques of selection of detail coefficients based on a threshold value are introduced by D. Donoho [35]. These techniques are known as soft thresholding and hard thresholding [35].

Assume $\left\{\beta_{j k}, j, k \in Z\right\}$ are detail coefficients of the noisy signal. In the hard thresholding one replaces $\beta_{j k}$ with $\hat{\beta}_{j k}$ defined by:

$\hat{\beta}_{j k}=\left\{\begin{array}{cc}\beta_{j k}, & \text { if }\left|\beta_{j k}\right| \geq T_{r} \\ 0, & \text { if }\left|\beta_{j k}\right|<T_{r}\end{array}\right.$

Where $T_{r}$ is a certain threshold. In the soft thresholding one replaces $\beta_{j k}$ with $\hat{\beta}_{j k}$ defined by: $\hat{\beta}_{j k}=\left\{\begin{array}{cl}\operatorname{sign}\left(\beta_{j k}\right)\left(\left|\beta_{j k}\right|-T_{r}\right), & \text { if }\left|\beta_{j k}\right| \geq T_{r} \\ 0, & \text { if }\left|\beta_{j k}\right|<T_{r}\end{array}\right.$

The value of the threshold $T_{r}$ is based on some prior information that may exist on the signal. A fixed threshold $T_{r}$, is introduced by Donoho and Johnstone [36] in the form:

$T_{r}=\sigma \sqrt{2 \log (n)}$

Where ${ }^{\sigma}$ is estimated using the median of the absolute deviation of the detail coefficients of the first wavelet decomposition of the signal, and ${ }^{n}$ is their number:

$\sigma=1.483 \times$ median $\left[\left(\beta_{J-1, k}\right) k \in Z\right]$

Let $J$ be the highest resolution level of the signal. Steps of high frequency noise cancellation using soft thresholding are:

- Do the first decomposition of the ECG signal $^{x(t)}$.

$x(t)=\sum_{k=0}^{2^{J-1}} \alpha_{J k} \phi_{J k}(t)$

First decomposition of ${ }^{x(t)}$ gives:

$x(t)=\sum_{k=0}^{2^{J-1}-1} \alpha_{J-1, k} \phi_{J-1, k}(t)+\sum_{k=0}^{{ }^{J-1}-1} \beta_{J-1, k} \psi_{J-1, k}(t)$

Compute ${ }^{\sigma}$ from $\beta_{J-1, k}, k \in Z$ and $T_{r}$ from equation (3).

- Compute the complete discrete wavelet decomposition of the signal. The expression of $x(t)$ becomes:

$x(t)=\alpha_{00} \phi_{00}(t)+\sum_{j=0}^{I} \sum_{k=0}^{2^{j}-1} \beta_{j k} \psi_{j k}(t)$

Replace all the detail coefficients $\beta_{j k}$ with $\hat{\beta}_{j k}$ (Equation 2). We obtain the new signal $\hat{x}(t)$ :

$\widehat{x}(t)=\alpha_{00} \phi_{00}(t)+\sum_{j=0}^{I} \sum_{k=0}^{2^{j}-1} \hat{\beta}_{j k} \psi_{j k}(t)$

Compute the inverse discrete wavelet transform of $\hat{x}(t)$ to obtain the denoised signal corresponding to the original signal $x(t)$ :

$\widehat{x}(t)=\sum_{k=0}^{2^{J}-1} \hat{\alpha}_{J k} \phi_{J k}(t)$

\subsection{Segmentation and feature extraction of heart beat}

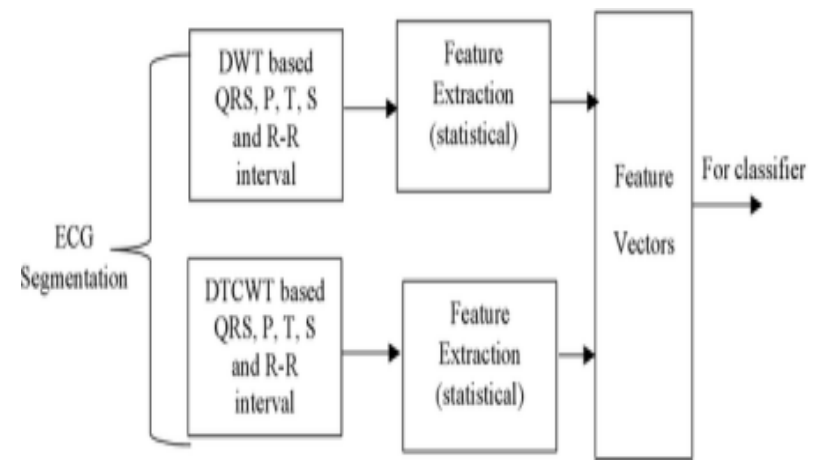

Fig. 4: ECG Segmentation Flow and Features Combination for Classification.

\subsubsection{Detection of the QRS complex}

To perform an automatic analysis of the ECG signal, detection of QRS complexes is a very important step. The detection of QRS complexes can be accomplished by a simple thresholding of the signal since the $\mathrm{R}$ waves are generally larger than the other waves in terms of amplitudes. But sometimes, in some cases, the amplitude 
of the $\mathrm{T}$ wave is comparable to that of the $\mathrm{R}$ wave, which could induce one of the errors in the end result of detection.

Moreover, the $\mathrm{R}$ wave may sometimes have a small amplitude and a very variable morphology from one cardiac cycle to another. Thus, good detection of QRS complexes is essential. This therefore requires a very adequate signal processing due to the difficulties encountered.

\subsubsection{Principle of the QRS complex detection algorithm}

The evolution of the power of digital processing tools led to the design and implementation of a variety of algorithms dedicated to the automatic detection of QRS complexes.

Thus, the detection of the QRS complex has been the subject of much work and continues to be a highly developed field of research The ECG signal from the pre-processing stage where it is subjected to a filtering operation of the different noises which contaminates it is decomposed into a wavelet. The choice of the mother wavelet used is of paramount importance in wavelet applications. In reality there is no well-defined criterion for the selection of the mother wavelet and this choice depends very much on the nature of the application and differs from one application to another. Several studies on the wavelet ECG signal analysis found that the 'Daubechies' wavelet family and more particularly the ' $\mathrm{db} 4$ ' wavelet is best suited for processing of the ECG signal because its shape is similar to the QRS complex.

\subsubsection{Detection of the $Q R S$ complex and the $R$ wave}

Most of the energy in the QRS complex is between $3 \mathrm{~Hz}$ and $40 \mathrm{~Hz}$ [37]. In the Fourier Transform, the waves with frequencies at $3 \mathrm{~dB}$ indicate that most of the energy of the QRS complex is located between the scales $2^{3}$ and $2^{5}$. From the scale $2^{4}$ the energy decreases. The detection of the QRS complex is based on the maximum modulus of the Discrete Wavelet Transformation (Multi Resolution Decomposition - MRD). The reason is that the maximum DWT modulus and the zero crossing correspond to the edges of the maximum frequency endpoints in the vicinity of the QRS complex.

\subsubsection{R-wave detection}

The $\mathrm{R}$ wave is the highest amplitude point of the possible threshold points found in the ECG signal. The lower scales $\left(2^{1}-2^{4}, \mathrm{~m}=1-4\right)$ represent the components of high frequencies, while the higher scales represent the low frequency components of the ECG signals [38].

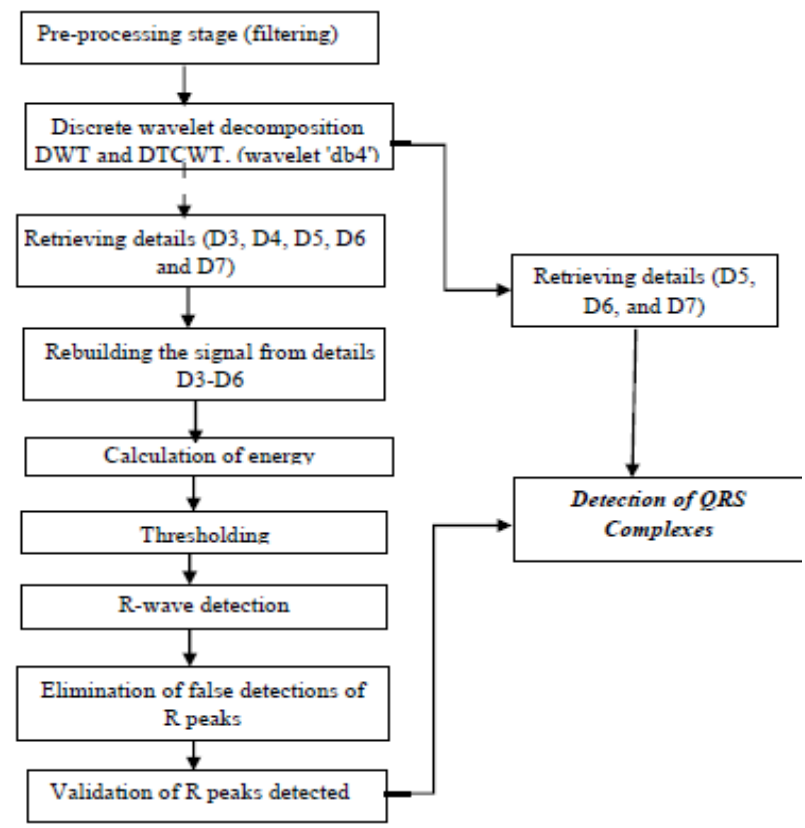

Fig. 5: Block Diagram of the Principle Adopted in the QRS Complex Detection Algorithm.
Maximum energy of the QRS complex is concentrated in the decomposition levels $2^{3}-2^{5}$. This indicates that the Wavelet coefficients of the detail signals $d_{3}, d_{4}$ and $d_{5}$ serve to identify the QRS complex:

$e_{1}=d_{3}+d_{4}+d_{5}$

With this expression the QRS region is correctly configured, however, it is difficult to identify the R-peak because of its oscillatory nature.

In order to remedy this effect, the function $e_{2}$ is defined as follows:

$e_{2}=\frac{d_{4}}{2^{n}}\left(d_{3}+d_{5}\right)$

Where ${ }^{n}$ is the decomposition level. The product module of the signals $e_{1 \text { and }} e_{2}$ is considered:

$e_{3}=\left|e_{1} \times e_{2}\right|$

The accuracy of the work of the extraction of characteristics depends mainly on the accuracy of the identification of the $\mathrm{R}$ wave, and for this the method of thresholding is used.

\subsubsection{Method of thresholding}

In this method the detail coefficients $\mathrm{d} 4$ are used to perform the detection of the R-wave. For this purpose, a lower limit is practically applied to eliminate the peaks that appear due to noise.

A strong threshold value is applied so that samples that are below the benchmark threshold are set to zero. Following is the formulation of threshold:

$\lambda=15 \% \times \max \left(d_{4}\right)$

$d_{4}(i)= \begin{cases}d_{4}(i) & d_{4}(i) \geq \lambda \\ 0 & d_{4}(i)<\lambda\end{cases}$

Next, the points Q and S are detected.

\subsubsection{Detection of $Q$ and $S$ points}

The points $\mathrm{Q}$ and $\mathrm{S}$ occur around the point $\mathrm{Q}$ around 0.1 seconds. Determining the distance from the zero point or near zero to the left of the point $\mathrm{Q}$ within a threshold threshold value provides the point Q.

Once the $\mathrm{R}$ points are detected, the $\mathrm{Q}$ and $\mathrm{S}$ points are identified to complete the complete detection of the QRS complex. Generally the $\mathrm{Q}$ and $\mathrm{S}$ waves have high frequencies and low amplitude, so their energies are mainly found in the lower scales in the Multiresolution analysis. Thus, the decomposition coefficients of the scales $d_{2}$ to $d_{5}$ will be used.

Since the points $\mathrm{Q}$ and $\mathrm{S}$ are inflection points on both sides of the point $R$, the first zero slope on both sides of the point $R$ provides the points $\mathrm{Q}$ and $\mathrm{S}$.

\subsubsection{Detection of $P$ and $T$ waves}

Once the QRS complexes have been identified, the identification of the $\mathrm{P}$ and $\mathrm{T}$ waves is made. For this purpose, the $R-R$ intervals are defined, which define a relative window between heartbeat and heartbeat, determining the heart rate. The local maximums of the signal $\left|d_{4}\right|=\left|W_{2^{4}} E C G[n]\right|$ are searched within this relative window, since the $\mathrm{P}$ and $\mathrm{T}$ waves are considered to be present in the R-R range if at least two of the local maxima exceed a threshold value that is proportional to the mean square root (RMS) of the WT measured between two consecutive QRS complexes. 


\subsubsection{Segmentation with dual tree complex wavelet transform} (DT-CWT)

Dual tree complex wavelet transform is implemented using two filter sets. Primary filter set presents at level 1 and the secondary set of filters exists at higher levels. There exists even length for the filters beyond the level 1 along with a group delay of about $1 / 4$. The time inversion of tree and filters in $b$ tree provides the remaining delay dissimilarity of $1 / 2$ samples. Accordingly, it is clear that two real DWTs are utilized in dual-tree complex wavelet transform and the complex coefficients are achieved using the consolidation of two trees. The energy discrepancy of complex wavelet coefficients is lower than that of DWT coefficients. Therefore, the DT-CWT outperforms the DWT for pattern recognition problems. The procedure for feature extraction using DT-CWT is described as follows:

- A window of 256 samples around R-vertex (out of which 128 from left and 128 from right) is selected for the extraction of QRS complex signal.

- Using the decomposition of QRS complex signal, perform 1D DTCWT up to 5 scales

- Detailed coefficients of fourth and fifth scale are selected as features. It is noteworthy that the upper tree of DTCWT delivers the coefficients equivalent to the real part whereas the lower tree of DTCWT delivers the imaginary part. Using these real and imaginary parts, the absolute values for the detail coefficients are calculated.

\subsubsection{Features extracted}

The seven standardized measurable esteems extricated from every wavelet sub-band can be registered by the accompanying conditions: Subsequent formulations represents outcome obtained from each wavelet sub-band:

Variance of QRS complex achieved by DWT and DTCWT methods:

Variance $(V)=\sum_{i=1}^{L-1}\left(z_{i}-m\right)^{2} p\left(z_{i}\right) /(L-1)^{2}$

Energy of P and T waves and QRS complex achieved by DWT and DTCWT methods:

$$
\begin{aligned}
& \operatorname{Energy}\left(E_{n}\right)=\sum_{i=0}^{L-1}\left(z_{i}-m\right)^{3} p\left(z_{i}\right) /(L-1)^{2} \\
& \text { uniformity }(U)=\sum_{i=0}^{L-1} p^{2}\left(z_{i}\right)
\end{aligned}
$$

$$
\operatorname{Entropy}\left(E_{v}\right)=-\sum_{i=0}^{L-1} p\left(z_{i}\right) \log _{2} p\left(z_{i}\right)
$$

Here, ${ }^{z_{i}}$ is a signals discrete transformed value.

$p\left(z_{i}\right)$ is probability of occurrence of discrete transformed values. $i=0,1,2 \ldots . L-1$ is the discrete levels.

Where $m=\sum_{i=0}^{L-1} z_{i} p\left(z_{i}\right)$ is the mean

Kurtosis of QRS complex signal, Q wave and T wave Kurt $\left(z_{i}\right)=E\left|\left(z_{i}-\mu\right)^{4}\right| / \sigma^{4}$.

\subsection{Classification using neural network classifier}

The choice of the classifier is very important to automatically classify the heartbeat..The idea of computation Recursive, parallel and fast during the learning phase is retained by the neuronal connectionist approach. The supervised networks are more appropriate for very complex separation surfaces because of their local learning. The ANN are generally associated with an algorithm called "learning" capable of modifying more or less automatically the processing performed of a given task.

In specific circumstances at the output of BPNN resembles Bayesian Posterior Probabilities . To get low error performance for given set of features along with selection of parameters such as training samples, hidden layer nodes and learning rate Bayesian Posterior Probabilities conditions are essential. The theoretical computation of number of hidden nodes is not a specific process for hidden layers. Testing method is commonly entertained for selection of these followed in the constrained environment of performance [39]. For $\mathrm{W}$ number of weights and $\mathrm{N}$ number of nodes, numbers of samples (m) are depicted to correctly classify future samples in following manner:

$m \geq O\left(\frac{W}{E} \log \frac{N}{E}\right)$

\section{Experimental setup}

\subsection{Database 1: MIT-BIH normal sinus rhythm database}

It provides 18 long ECG records duration of 18 patients (5 men and 13 women) who did not present no significant arrhythmia type. The time or velocity is measured on the horizontal axis and the amplitude or voltage in the vertical axis. The shape of the ECG wave signifies the purpose of the conduction system of the heart, which generally initiates and conducts electrical activity. The electrocardiographic tracing is composed of several components or waves, which include the following: $\mathrm{P}$ wave, QRS complex, T wave, ST segment, interval P-R and maybe the U wave.

The $\mathrm{P}$ wave corresponds to depolarization of the atrial muscle. Usually has a peak value less than $0.25 \mathrm{mV}$ and $0.11 \mathrm{~s}$ or less in duration. The first negative deflection after the above wave is the $\mathrm{Q}$ wave, which lasts less than $0.03 \mathrm{~s}$ and covers less than $25 \%$ of the amplitude of the R-wave. The first positive deflection posterior to the $\mathrm{P}$ wave is the $\mathrm{R}$ wave, and the $\mathrm{S}$ wave is the first deflection negative after the $\mathrm{R}$ wave.

The QRS complex, which begins the $\mathrm{Q}$ wave, or the $\mathrm{R}$ wave if there is no $\mathrm{Q}$ wave, and ends with the $\mathrm{S}$ wave, represents depolarization of the ventricular muscle. The complex QRS usually lasts from 0.04 to $0.10 \mathrm{~s}$.

The $\mathrm{T}$ wave corresponds to repolarization of the ventricular muscle. Appears after the QRS complex and usually has the same deflection. If identified, the $\mathrm{U}$ wave appears after the $\mathrm{T}$ wave. The $\mathrm{U}$ wave is believed to represent repolarization of the Purkinje fibres. When it appears, it follows the $\mathrm{T}$ wave and has approximately the same size as the $\mathrm{P}$ wave, and may be mistaken for a $\mathrm{P}$ wave additional.

The Q-T interval represents the total repolarization and depolarization time ventricular, and measured from the beginning of the $\mathrm{Q}$ wave, or from the $\mathrm{R}$ wave if there is no wave $\mathrm{Q}$, at the end of the $\mathrm{T}$ wave. It varies with heart rate, and lasts from 0.32 to $0.40 \mathrm{~s}$ if heart rate is 65 to 95 beats per minute.

The amplitude and duration of a normal sinus rhythm of the ECG signal are indicated in Table 1 [40].

Table 1: Characteristics of the ECG Signal

\begin{tabular}{llll}
\hline Amplitude & & Interval & \\
\hline P wave & $0.25 \mathrm{mV}$ & P-R & 0.12 to $0.20 \mathrm{~s}$ \\
R Wave & $1.60 \mathrm{mV}$ & O-T & 0.35 to $0.44 \mathrm{~s}$ \\
O Wave & $25 \%$ R wave & ST & 0.05 to $0.15 \mathrm{~s}$ \\
T Wave & 0.1 to $0.5 \mathrm{mV}$ & ORS & $0.09 \mathrm{~s}$ \\
& & P & $0.11 \mathrm{~s}$ \\
\hline
\end{tabular}

As described in database, the electrical activity of the heart is monitored by certain electrodes at points located on the surface of the skin. The electrical signal is very low, usually between $0.1 \mathrm{mV}$ and $1.6 \mathrm{mV}$. These signals are found in the spectrum of frequencies ranging from $0.05 \mathrm{~Hz}$ to frequency of $100 \mathrm{~Hz}$. As recorded in previous studies it is considered that the rhythm is normal sinus in following cases:

- All P waves are followed by QRS complexes.

- Regular rhythm (P-P intervals have variations less than 10\%).

- Frequency between 60 and 100 bpm.

- The P wave is positive in I, II, aVF, V4, V5 and V6 and negative in aVR.

- The PR intervals are constant and less than $0.20 \mathrm{~s}$. 


\subsection{Segmentation result}

The analysis was performed for detection of the QRS complex, 13 ECG signals from the MIT-BIH database Normal Sinus Rhythm Database (nsrdb) [41] with a duration of 3600 seconds each electrocardiographic record has been taken. The results obtained in the identification of QRS complexes of different patients with ECG signals with different morphologies. The precision achieved with this DWT algorithm is $98 \%$ with a computational cost Very low.

Table 2: QRS Complex Identified with the DWT Algorithm

\begin{tabular}{|c|c|c|c|c|}
\hline No. & ECG Signal & QRS Complexes & QRS Detected & Percentage \\
\hline 1 & nsrdb $/ 16265 \mathrm{~m}$ & 5281 & 5237 & 98.01 \\
\hline 2 & $\mathrm{nsrdb} / 16272 \mathrm{~m}$ & 3736 & 3431 & 89.14 \\
\hline 3 & $\mathrm{nsrdb} / 16273 \mathrm{~m}$ & 4976 & 4932 & 99.10 \\
\hline 4 & $\mathrm{nsrdb} / 16420 \mathrm{~m}$ & 5907 & 5500 & 92.11 \\
\hline 5 & $\begin{array}{l}\text { nsrdb } / 161483 \\
\mathrm{~m}\end{array}$ & 5481 & 5431 & 98.19 \\
\hline 6 & $\mathrm{nsrdb} / 16539 \mathrm{~m}$ & 5247 & 5134 & 96.15 \\
\hline 7 & $\mathrm{nsrdb} / 16773 \mathrm{~m}$ & 4424 & 4402 & 99.34 \\
\hline 8 & $\mathrm{nsrdb} / 16786 \mathrm{~m}$ & 4406 & 4385 & 99.43 \\
\hline 9 & $\mathrm{nsrdb} / 16795 \mathrm{~m}$ & 4112 & 4016 & 97.45 \\
\hline 10 & $\mathrm{nsrdb} / 17052 \mathrm{~m}$ & 4463 & 4450 & 99.52 \\
\hline 11 & $\mathrm{nsrdb} / 17453 \mathrm{~m}$ & 4963 & 4894 & 97.87 \\
\hline 12 & $\mathrm{nsrdb} / 18177 \mathrm{~m}$ & 5832 & 5458 & 91.39 \\
\hline \multirow[t]{2}{*}{13} & $\mathrm{nsrdb} / 18184 \mathrm{~m}$ & 5208 & 5191 & 99.04 \\
\hline & Total QRS & 64036 & 62690 & $96.89 \%$ \\
\hline
\end{tabular}

Table 3: QRS Complexes Identified with the DT-CWT Algorithm

\begin{tabular}{|c|c|c|c|c|}
\hline No. & ECG Signal & QRS Complexes & QRS Detected & Percentage \\
\hline 1 & $\mathrm{nsrdb} / 16265 \mathrm{~m}$ & 5281 & 5267 & 99.73 \\
\hline 2 & $\mathrm{nsrdb} / 16272 \mathrm{~m}$ & 3736 & 3461 & 92.64 \\
\hline 3 & $\mathrm{nsrdb} / 16273 \mathrm{~m}$ & 4976 & 4941 & 99.30 \\
\hline 4 & $\mathrm{nsrdb} / 16420 \mathrm{~m}$ & 5907 & 5565 & 94.21 \\
\hline 5 & $\mathrm{nsrdb} / 161483 \mathrm{~m}$ & 5481 & 5441 & 99.27 \\
\hline 6 & $\mathrm{nsrdb} / 16539 \mathrm{~m}$ & 5247 & 5134 & 97.85 \\
\hline 7 & $\mathrm{nsrdb} / 16773 \mathrm{~m}$ & 4424 & 4412 & 99.73 \\
\hline 8 & $\mathrm{nsrdb} / 16786 \mathrm{~m}$ & 4406 & 4405 & 99.98 \\
\hline 9 & $\mathrm{nsrdb} / 16795 \mathrm{~m}$ & 4112 & 4066 & 98.88 \\
\hline 10 & $\mathrm{nsrdb} / 17052 \mathrm{~m}$ & 4463 & 4455 & 99.82 \\
\hline 11 & $\mathrm{nsrdb} / 17453 \mathrm{~m}$ & 4963 & 4894 & 98.61 \\
\hline 12 & $\mathrm{nsrdb} / 18177 \mathrm{~m}$ & 5832 & 5458 & 93.59 \\
\hline \multirow[t]{2}{*}{13} & $\mathrm{nsrdb} / 18184 \mathrm{~m}$ & 5208 & 5191 & 99.67 \\
\hline & Total QRS & 64036 & 62690 & $98 \%$ \\
\hline
\end{tabular}

\subsection{Algorithm of the estimation of the heart rate}

Once the R points of the different ECG signals have been identified, the cardiac frequency of each patient has been estimated by taking the times in which each $61 \mathrm{R}$ points, corresponding to the time elapsed in 60 beats ( $60 \mathrm{R}-\mathrm{R}$ intervals), are produced. In this way the frequency has been measured every 61 beats along the entire signal (3600 seconds) with the following formula:

$$
\text { bpm }=\frac{1}{\frac{t_{2}-t_{1}}{60}} \times 60=\frac{3600}{t_{2}-t_{1}}
$$

\section{$t_{1} \quad$ First $R$ wave Time}

$\begin{cases}t_{1} & \\ t_{2} & 61^{\text {st }} R \text { wave Time }\end{cases}$

Once the determination of the cardiac frequencies was performed, Table 4 shows the minimum, maximum, median and standard deviation values detected, as well as the mean value of the frequencies of each patient over an hour, obtained by the algorithm.

\subsection{Database 2: MIT-BIH atrial fibrillation database (AFDB)}

It includes 25 long-term ECG records corresponding to patients who present episodes of atrial fibrillation. The records in the database last approximately 10 hours and [2] electrocardiographic signals sampled at $250 \mathrm{~Hz}$ with a resolution of 12 bits and a range of $\leq 10 \mathrm{mV}$. All signals are labelled.

\subsection{Database 3: MIT-BIH supraventricular arrhythmia database (SVDB)}

These are provided 78 half-hour records that have been specially selected to complement the examples of supraventricular arrhythmia provided in the MIT-BIH Arrhythmia Database.

Table 4: Cardiac Frequencies Identified with the DWT Algorithm

\begin{tabular}{|c|c|c|c|c|c|c|}
\hline \multirow[t]{2}{*}{ No. } & \multirow{2}{*}{$\begin{array}{l}\text { ECG } \\
\text { Signal }\end{array}$} & \multicolumn{4}{|c|}{ Beats/Minute } & \multirow[t]{2}{*}{$\sigma$} \\
\hline & & $\min$ & $\max$ & half & median & \\
\hline 1 & $\begin{array}{l}\text { nsrdb } \\
16265 \mathrm{~m}\end{array}$ & 79 & 121 & 89 & 86 & 8.85 \\
\hline 2 & $\begin{array}{l}\text { nsrdb } \\
16272 \mathrm{~m}\end{array}$ & 50 & 89 & 67 & 59 & 12.95 \\
\hline 3 & $\begin{array}{l}\text { nsrdb } \\
16273 \mathrm{~m}\end{array}$ & 69 & 115 & 84 & 82 & 10.18 \\
\hline 4 & $\begin{array}{l}\text { nsrdb } \\
16420 \mathrm{~m}\end{array}$ & 69 & 118 & 94 & 95 & 10.99 \\
\hline 5 & $\begin{array}{l}\text { nsrdb } \\
16483 \mathrm{~m}\end{array}$ & 80 & 102 & 91 & 92 & 4.36 \\
\hline 6 & $\begin{array}{l}\text { nsrdb } \\
16539 \mathrm{~m}\end{array}$ & 71 & 117 & 87 & 83 & 12.38 \\
\hline 7 & $\begin{array}{l}\text { nsrdb } \\
16773 \mathrm{~m}\end{array}$ & 64 & 114 & 80 & 74 & 13.53 \\
\hline 8 & $\begin{array}{l}\text { nsrdb } \\
16786 \mathrm{~m}\end{array}$ & 69 & 114 & 79 & 73 & 11.73 \\
\hline 9 & $\begin{array}{l}\text { nsrdb } \\
16795 \mathrm{~m}\end{array}$ & 53 & 114 & 76 & 71 & 15.99 \\
\hline 10 & $\begin{array}{l}\text { nsrdb } \\
17052 \mathrm{~m}\end{array}$ & 57 & 114 & 80 & 78 & 13.77 \\
\hline 11 & $\begin{array}{l}\text { nsrdb } \\
17453 \mathrm{~m}\end{array}$ & 68 & 108 & 84 & 83 & 7.65 \\
\hline 12 & $\begin{array}{l}\text { nsrdb } \\
18177 \mathrm{~m}\end{array}$ & 76 & 112 & 92 & 90 & 8.47 \\
\hline 13 & $\begin{array}{l}\text { nsrdb } \\
18184 \mathrm{~m}\end{array}$ & 74 & 111 & 88 & 86 & 10.02 \\
\hline
\end{tabular}

4.6. Database 4: MIT-BIH malignant ventricular arrhythmia database (VFDB)

MIT-BIH Malignant Ventricular Arrhythmia Database. With 22 media records duration of patients who have had episodes of sustained ventricular tachycardia, ventricular flutter, and ventricular fibrillation.

In this case, the signals corresponding to patients with arrhythmias cardiac disorders. Arrhythmias comprise all alterations in frequency, rhythm or both parameters. They originate in the conduction system, and not in the myocardium nor in their structures themselves and are identified when analyzing the electrocardiographic tracings. The arrhythmias of the Wavelet analysis are catagorized into two types:

\subsubsection{Ventricular arrhythmias}

- Ventricular tachycardias (VT-ventricular tachycardia),

- Ventricular fibrillation (VF-ventricular fibrillation),

- Ventricular flutter (VFL-ventricular flutter)

\subsubsection{Supraventricular arrhythmias}

- Supraventricular Tachyarrhythmia (SVTA)

- Atrial fibrillation (AF)

\subsubsection{Ventricular arrhythmias}

To obtain ECG signals to. Ventricular arrhythmias In the processing of ventricular arrhythmias, a total of 23 ECG Records Database MIT-BIH Malignant Ventricular Arrhythmia Database (vfdb) [41] of 35 minutes each, with a frequency sampling rate of $\mathrm{f}=250 \mathrm{~Hz}$, belonging to patients who presented episodes of sustained ventricular tachycardia (VT), ventricular fibrillation (VF) and episodes of Supraventricular Tachyarrhythmia (SVTA). Table 5 shows the signals with the different episodes of Ventricular Tachycardia (VT) and Ventricular Fibrillation (VF) recorded on each of the ECG signals. 
Table 5: Signals with the Different Episodes of Ventricular Tachycardia (VT) and Ventricular Fibrillation (VF) Recorded on Each of the ECG

\begin{tabular}{cccc}
\hline ECG Signal & VT & VF & VFL \\
\hline Vfdb-420 & 3 & - & - \\
Vfdb-421 & 50 & - & - \\
Vfdb-422 & 2 & 3 & - \\
Vfdb-423 & 1 & - & - \\
Vfdb-424 & - & 1 & 1 \\
Vfdb-425 & 4 & - & - \\
Vfdb-426 & 1 & 3 & - \\
Vfdb-427 & - & - & - \\
Vfdb-428 & 3 & - & - \\
Vfdb-429 & 3 & - & 2 \\
Vfdb-430 & 6 & 6 & 1 \\
Vfdb-602 & - & - & - \\
Vfdb-607 & - & - & - \\
Vfdb-615 & & & - \\
Vfdb-611 & & & - \\
Vfdb-418 & - & - & 12 \\
Vfdb-419 & - & - & 12 \\
Total & $\mathbf{s s}$ & $\mathbf{1 3}$ & $\mathbf{2 8}$ \\
\hline
\end{tabular}

\subsubsection{Supraventricular arrhythmias}

The treatment of supraventricular arrhythmias has required 6 records of the Database MIT-BIH Supraventricular Arrhythmia Database (svdb). See the Table 6 with the signs along with the episodes of Supraventricular Tachycardia (SVTA) annotated in the Database. In the Wavelet analysis of atrial fibrillation (AF) episodes, processed a total of 13 signals: (a) 2 signals from the MIT-BIH Database Malignant Ventricular Arrhythmia Database (vfdb), and (b) 11 signals from the MIT-BIH Atrial Fibrillation Database (afdb). 21 $\mathrm{AF}$ episodes, shown in Table 6 , corresponding to the processed ECG signals.

Table 6: Signals with the Different Episodes of Supraventricular Tachycardia (SVVT) and Atrial Fibrillation (AF)

\begin{tabular}{ccc}
\hline ECG Signals & SVTA & AF \\
\hline Svdb-801 & 45 & - \\
Svdb-806 & 12 & - \\
Svdb-807 & 15 & - \\
Svdb-808 & 19 & - \\
Svdb-827 & 4 & - \\
Svdb-887 & 16 & - \\
Vfdb-423 & - & 2 \\
Vfdb-615 & - & 6 \\
Afdb-04043 & - & 2 \\
Afdb-04126 & - & 6 \\
Afdb-04048 & - & 1 \\
Afdb-04126 & - & 2 \\
Afdb-04936 & - & 4 \\
Afdb-06426 & - & 1 \\
Afdb-06413 & - & 3 \\
Afdb-07162 & - & 2 \\
Afdb-07859 & - & 1 \\
Afdb-07869 & - & 1 \\
\hline
\end{tabular}



Fig. 6: ECG of 418 for Ventricular Flutter (VFL).

The main results of the VFL analysis are outlined.

1) The QRS complexes are not identified in the scales of energies, because it not clearly defined in the signal.

2) The web is clearly seen in the frame with higher frequencies at approximately $3 \mathrm{~Hz}$, corresponding to the cardiac frequency greater than $180 \mathrm{bpm}$.

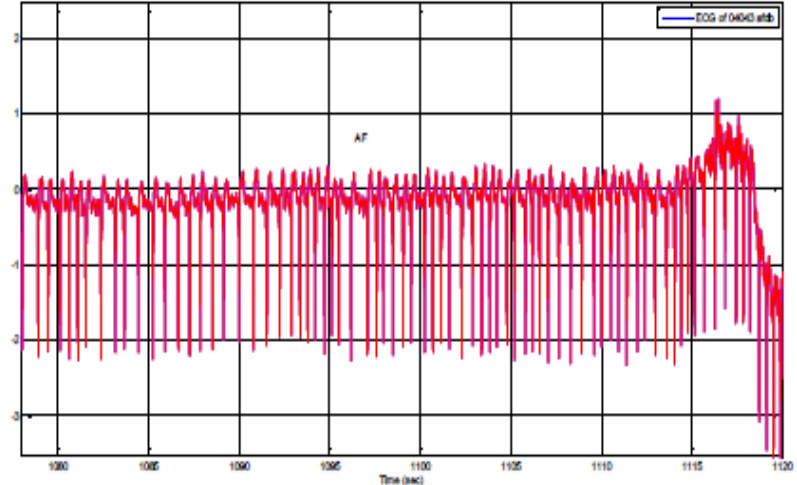

Fig. 7: ECG of 04043 for Atrial Fibrillation (AF)

The rhythms observed in the signals show quite irregularity, with high heart rates, higher than $200 \mathrm{bpm}$. It is verified that in many episodes with VF the absence of the $\mathrm{P}$ wave can colloborated with the union of two QRS complexes with a certain amount of energy Wavelet.

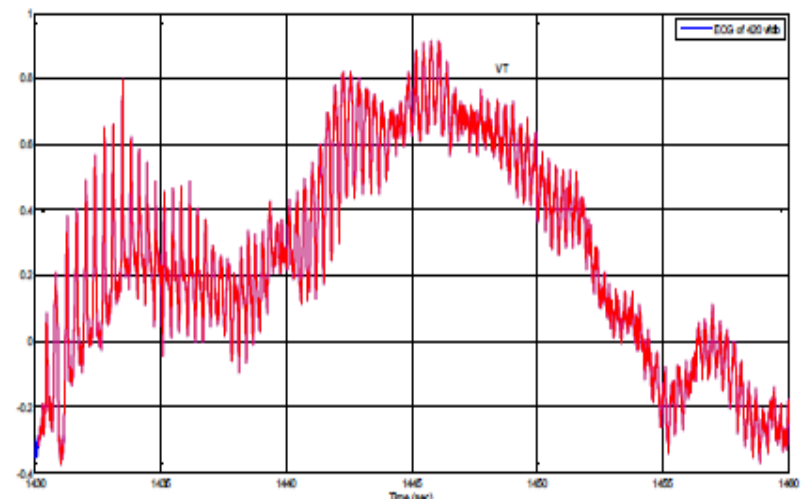

Fig. 8: ECG of 420 for Ventricular Tachycardia (VT).

1) In VT episodes the rhythm appears, usually regular.

2) The $P$ wave is not seen, neither in the time graph nor in the Wavelet scalogram time-frequency.

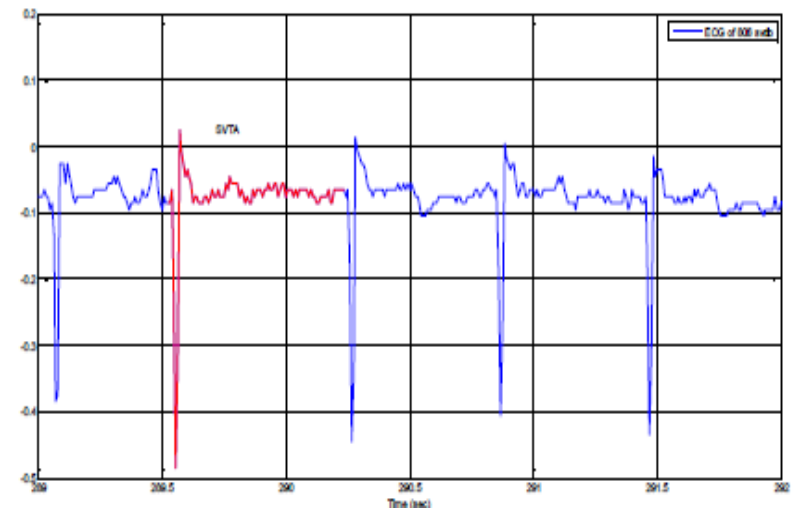

Fig. 9: ECG of 808 for Supraventricular Tachyarrhythmia (SVTA).

It identifies in the scales a signal with a certain regular rhythm, recognizable by the frequency band, around the $2.1 \mathrm{~Hz}-3.6 \mathrm{~Hz}$ 


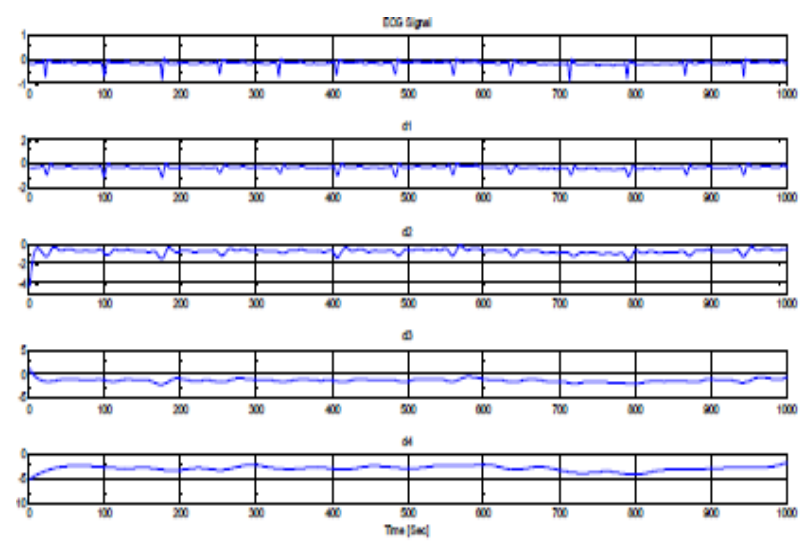

Fig. 10: ECG Signal after Wavelet Decomposition.

\subsection{Implementation with neural network}

The ventricular and supraventricular arrhythmias samples are taken for training and testing. There

are 6 records of svdb ,13 signals from af, 21 signals of afdb and 6 signals from vfdb with duration of 10 hours has been considered. 1 hour samples (approximately 20034 samples of signal) has been processed for training.After getting all the extracted features, discussed above is trained. Testing is performed on (60000 samples) .It compared with ground truth label and found accuracy $99 \%$.

\section{Conclusion}

Wavelet analysis performed on ECG signals with normal sinus rhythm with DWT and DTCWT has allowed us to identify graphically (wavelet energy in 2 D) characteristics of the heartbeat: complex QRS, P, S and T waves, as well as heart rate. Noise elimination techniques have been used in ECG signals with Wavelet (thresholding) decomposing signals with and without added noise with the decomposition algorithm (MRA) using daubechies Wavelet, and applying the universal threshold (soft) with scaled white noise structure (scaled white noise). The algorithm designed in this Paper, based on the DWT and DTCWT for the detection of the QRS complex, and the $\mathrm{P}$ and $\mathrm{T}$ waves, .It is further compared with ground truth ,DTCWT has offered high accuracy ,overall experimental analysis claims higher accuracy as an average $98 \%$. The ventricular and supraventricular arrhythmias studied with the DWT and DTCWT with various feature extraction after transform, further those features are trained with neural network to justify the fully automatic classification for arrhythmias analysis. Our work has taken care of optimal feature extraction methods after ECG segmentation and it is considered as the feature vector for training to neural network. The evaluated results claims better accuracy with NN approximately 99\% compared with ground truth (Extracted from database).

\section{References}

[1] K.V.L.Narayana, and A.B .Rao, "Wavelet based QRS detection in ECG using MATLAB" in Innovative Systems Design and Engineering, vol. 2, no.7, pp.60-69, November 2011.

[2] S. Kiranyaz, T. Ince, J. Pulkkinen, and M. Gabbouj," Personalized long-term ECG classification: A systematic approach" in Expert Systems with Applications, vol.38, no.4, pp.3220-3226, April 2011

[3] https://www.slideshare.net/usamaelsayed2/ecg-interpretation-byusama-elsayed

[4] C. Saritha,, V. Sukanya, and Y.N. Murthy,, " ECG signal analysis using wavelet transforms", in Bulg. J. Phys, vol. 35, no.1, pp.68-77, February 2008.

[5] T. Xu, Z. Yang, M. Vatta, A. Rampazzo, G. Beffagna, K. Pillichou, S.E. Scherer, J. Saffitz, J. Kravitz, W. Zareba, and G.A. Danieli, "Compound and digenic heterozygosity contributes to arrhythmogenic right ventricular cardiomyopathy" in Journal of the American College of Cardiology, vol. 55, no. 6, pp.587-597, February 2010. https://doi.org/10.1016/j.jacc.2009.11.020.
[6] G.S. Kamath, W. Zareba, J. Delaney, J.N. Koneru, W. McKenna, K. Gear, S. Polonsky, D. Sherrill, , D. Bluemke, F. Marcus, and J.S. Steinberg," "Value of the signal-averaged electrocardiogram in arrhythmogenic right ventricular cardiomyopathy/dysplasia", in Heart Rhythm, vol. 8, no. 2, pp.256-262. February 2011. https://doi.org/10.1016/j.hrthm.2010.10.007.

[7] S. Saadi, M. Bettayeb, A. Guessoum, and M.K. Abdelhafidi," Artificial bee colony optimized neural network model for ecg signals classification" in International Conference on Neural Information Processing , pp. 339-346. Springer, Berlin, Heidelberg, November 2012. https://doi.org/10.1007/978-3-642-34478-7_42.

[8] D.G. Katritsis, G.C. Siontis and A.J. Camm," Prognostic significance of ambulatory ECG monitoring for ventricular arrhythmias" in Progress in cardiovascular diseases, vol. 56, no. 2, pp.133-142, September 2013.

[9] G. Koulaouzidis, S. Das, G. Cappiello, E.B. Mazomenos, K. Maharatna, P.E. Puddu, and J.M. Morgan, "Prompt and accurate diagnosis of ventricular arrhythmias with a novel index based on phase space reconstruction of ECG", in International journal of cardiology, 182, pp.38-43, April 2015.

[10] C.C. Lin and C.M. Yang, " Heartbeat Classification Using Normalized RR Intervals and Wavelet Features" in Computer, Consumer and Control (IS3C), 2014 International Symposium on IEEE, pp. 650-653, June 2014.

[11] C.C. Lin and C.M. Yang, "Heartbeat classification using normalized RR intervals and morphological features" in Mathematical Problems in Engineering, May 2014.

[12] A. Ebrahimzadeh, and A. Khazaee," Detection of premature ventricular contractions using MLP neural networks: A comparative study" in Measurement, vol 43, no. 1, pp.103-112, January 2010.

[13] M. Korürek, and B. Doğan, "ECG beat classification using particle swarm optimization and radial basis function neural network" in Expert systems with Applications, vol. 37, no. 12, pp.7563-7569,December 2010. https://doi.org/10.1016/j.eswa.2010.04.087.

[14] R.J. Martis, U.R. Acharya, and L.C. Min, "ECG beat classification using PCA, LDA, ICA and discrete wavelet transform" in Biomedical Signal Processing and Control, vol.8 no. 5, pp.437-448, January 2013. https://doi.org/10.1016/j.bspc.2013.01.005.

[15] Y. Kaya, and H. Pehlivan, "Classification of premature ventricular contraction in ECG “,in International Journal of Advanced Computer Science and Applications, vol. 6, no. 7, pp.34-40, April 2015.

[16] G.H. Kleisinger, L. Barbosa, and J.E. Monzón, "Neural-Network Detection of Abnormal Ventricular Beats Using Temporal and Morphological Features of the Electrocardiographic Signal", in Proceedings of the Fourth World Multiconference on Systemics, Cybernetics and Informatics, July 2000.

[17] V. Krasteva, and I. Jekova, "Assessment of ECG frequency and morphology parameters for automatic classification of life-threatening cardiac arrhythmias" in Physiological measurement, vol. 26, no. 5, p.707, October 2005. https://doi.org/10.1088/0967-3334/26/5/011.

[18] Y. Sun, K.L. Chan, and S.M. Krishnan, "Characteristic wave detection in ECG signal using morphological transform"' in BMC cardiovascular disorders, vol. 5, no.1 p.28 September 2005

[19] J.E. Monzon, and M.I. Pisarello, "Cardiac beat classification using a fuzzy inference system", In Engineering in Medicine and Biology Society, 2005. IEEE-EMBS 2005.27 ${ }^{\text {th }}$ Annual International Conference of the IEEE, pp. 5582-5584, September 2005. https://doi.org/10.1109/IEMBS.2005.1615750.

[20] M. Mohanty, P.K. Biswal, and S.K. Sabut, "Feature extraction of ECG signal for detection of ventricular fibrillation", In Man and Machine Interfacing (MAMI), 2015 International Conference, IEEE, pp. 1-5, December 2015. https://doi.org/10.1109/MAMI.2015.7456595.

[21] M.G. Tsipouras, D.I. Fotiadis, and D. Sideris, "An arrhythmia classification system based on the RR-interval signal", in Artificial intelligence in medicine, vol. 33, no. 3, pp.237-250, March 2005

[22] H. Gothwal, S. Kedawat and R. Kumar, "Cardiac arrhythmias detection in an ECG beat signal using fast fourier transform and artificial neural network" in Journal of Biomedical Science and Engineering, vol. 4, no. 04, p.289, May 2011. https://doi.org/10.4236/jbise.2011.44039.

[23] L. Li, F. Wang, T. Ban, S. Guo, and B. Gong, "Network flow classification based on the rhythm of packets", In Neural Information Processing, Springer Berlin/Heidelberg, pp. 45-52, November 2011.

[24] N. Kingsbury, "Complex wavelets for shift invariant analysis and filtering of signals"' in Applied and computational harmonic analysis vol. 10, no. 3, pp.234-253, May 2001.

[25] M. Thomas, M.K. Das, and S. Ari, "Classification of cardiac arrhythmias based on dual tree complex wavelet transform", in Communi- 
cations and Signal Processing (ICCSP), 2014 International Conference, IEEE, pp. 729-733. April 2014. https://doi.org/10.1109/ICCSP.2014.6949939.

[26] G. Chen, Automatic EEG seizure detection using dual-tree complex wavelet-Fourier features. Expert Systems with Applications, vol. 41, no. 5, pp.2391-2394 April 2014. https://doi.org/10.1016/j.eswa.2013.09.037.

[27] M. Thomas, M.K. Das, and S. Ari,"Automatic ECG arrhythmia classification using dual tree complex wavelet based features", in AEUInternational Journal of Electronics and Communications, vol. 69, no. 4 , pp.715-721, January 2015

[28] Y. Özbay, and G. Tezel, "A new method for classification of ECG arrhythmias using neural network with adaptive activation function", in Digital Signal Processing, vol. 20, no. 4, pp.1040-1049, July 2010. https://doi.org/10.1016/j.dsp.2009.10.016.

[29] S.M. Jadhav, S.L. Nalbalwar, and A. Ghatol, "Artificial neural network based cardiac arrhythmia classification using ECG signal data" In Electronics and Information Engineering (ICEIE), 2010 International Conference, IEEE ,Vol. 1, pp. V1-228. August 2010. https://doi.org/10.1109/ICEIE.2010.5559887.

[30] H. Gothwal, S. Kedawat and R. Kumar, "Cardiac arrhythmias detection in an ECG beat signal using fast fourier transform and artificial neural network" in Journal of Biomedical Science and Engineering, vol. 4, no.4, p.289 , April 2011 https://doi.org/10.4236/jbise.2011.44039.

[31] S.M. Jadhav, S.L. Nalbalwar and A.A. Ghatol, "Modular neural network based arrhythmia classification system using ECG signal data." In International Journal of Information Technology and Knowledge Management, vol. 4, no.1, pp.205-209, June 2011.

[32] M.K. Sarkaleh and A. Shahbahrami, "Classification of ECG arrhythmias using discrete wavelet transform and neural networks" in International Journal of Computer Science, Engineering and Applications, vol. 2, no. 1, p.1, February 2012.

[33] H.M. Rai, A. Trivedi, and S. Shukla, "ECG signal processing for abnormalities detection using multi-resolution wavelet transform and Artificial Neural Network classifier" in Measurement, vol. 46, no. 9 , pp.3238-3246, May 2013.

[34] P. Melin, J. Amezcua, F. Valdez, and O. Castillo, "A new neural network model based on the LVQ algorithm for multi-class classification of arrhythmias", in Information sciences, 279, pp.483-497, September 2014.

[35] D.L. Donoho, "De-noising by soft-thresholding" in IEEE trans. on information theory, vol. 41, no. 3, pp.613-627, May 1995. https://doi.org/10.1109/18.382009.

[36] D.L. Donoho, and I.M. Johnstone, "Adapting to unknown smoothness via wavelet shrinkage" in Journal of the american statistical association, vol. 90, no. 432, pp.1200-1224, February 1995.

[37] P. Sasikala and R.S.D. WahidaBanu, "Extraction of P wave and T wave in Electrocardiogram using Wavelet Transform", in International Journal of Computer Science and Information Technologies, vol. 2, no. 1, pp.489-493, March 2011.

[38] S. Behbahani, and N.J. Dabanloo, "Detection of QRS complexes in the ECG signal using multiresolution wavelet and thresholding method" in Computing in Cardiology, pp. 805-808. IEEE, September 2011.

[39] S.N. Yu and Y.H. Chen, "Electrocardiogram beat classification based on wavelet transformation and probabilistic neural network", in Pattern Recognition Letters, vol. 28, no. 10, pp.1142-1150, July 2007. https://doi.org/10.1016/j.patrec.2007.01.017.

[40] C. Saritha, , V. Sukanya and Y.N. Murthy, "ECG signal analysis using wavelet transforms" in Bulg. J. Phys, vol. 35, no. 1, pp.68-77 ,February 2008.

[41] AL. Goldberger, LAN Amaral, L. Glass, JM. Hausdorff, P.Ch. Ivanov, RG. Mark, JE. Mietus, GB. Moody, C-K Peng, HE. Stanley, PhysioBank, PhysioToolkit, and PhysioNet: Components of a New Research Resource for Complex Physiologic Signals. Circulation 101(23):e215-e220 [Circulation Electronic Pages; http://circ.ahajournals.org/content/101/23/e215.full] June 2000. 